\title{
Correction to: N-Myc promotes therapeutic resistance development of neuroendocrine prostate cancer by differentially regulating miR-421/ ATM pathway
}

Yu Yin ${ }^{1,2,3+}$, Lingfan Xu ${ }^{1,2+}$, Yan Chang ${ }^{2,4}$, Tao Zeng ${ }^{2,5}$, Xufeng Chen², Aifen Wang ${ }^{2}$, Jeff Groth ${ }^{2}$, Wen-Chi Foo ${ }^{2}$, Chaozhao Liang ${ }^{1 *}$, Hailiang $\mathrm{Hu}^{2,6^{*}}$ and Jiaoti Huang ${ }^{2,6,7^{*}}$

\section{Correction to: Mol Cancer}

$$
\text { https://doi.org/10.1186/s12943-019-0941-2 }
$$

Following publication of the original article [1], the authors reported that name that appeared in published online version is incorrect. Aifeng Wang should be Aifen Wang. Corrected name is provided in the author group section above.

\begin{abstract}
Author details
'Department of Urology, First Affilated Hospital of Anhui Medical University, Hefei 230022, China. ${ }^{2}$ Department of Pathology, Duke Unversity School of Medicine, DUMC box 103864, 905 S. Lasalle Street, Durham, NC 27710, USA. ${ }^{3}$ Department of Pathology, Anhui Medical University, Hefei 230032, China. ${ }^{4}$ Institute of Clinical Pharmacology, Anhui Medical University, Hefei, China. ${ }^{5}$ Department of Urology, Jiangxi Province People's Hospital, Nanchang, China. ${ }^{6}$ Duke Cancer Institute, Duke University School of Medicine, Durham, NC 27710, USA. ${ }^{7}$ Department of Pharmacology and Cancer Biology, Duke University School of Medicine, Durham, NC 27710, USA.
\end{abstract}

Published online: 19 June 2019

\section{Reference}

1. Yu Yin, Lingfan $\mathrm{Xu}$, Yan Chang, Tao Zeng, Xufeng Chen, Aifen Wang, Jeff Groth, Wen-Chi Foo, Chaozhao Liang, Hailiang Hu and Jiaoti Huang. N-Myc promotes therapeutic resistance development of neuroendocrine prostate cancer by differentially regulating miR-421/ ATM pathway.2019. https://doi. org/10.1186/s12943-019-0941-2

\footnotetext{
*Correspondence: liang_chaozhao@163.com; hailiang.hu@duke.edu; jiaoti.huang@duke.edu

${ }^{t}$ Yu Yin and Lingfan Xu contributed equally to this work.

'Department of Urology, First Affilated Hospital of Anhui Medical University, Hefei 230022, China

${ }^{2}$ Department of Pathology, Duke Unversity School of Medicine, DUMC box

103864, 905 S. Lasalle Street, Durham, NC 27710, USA

Full list of author information is available at the end of the article
}

(c) The Author(s). 2019 Open Access This article is distributed under the terms of the Creative Commons Attribution 4.0 International License (http://creativecommons.org/licenses/by/4.0/), which permits unrestricted use, distribution, and reproduction in any medium, provided you give appropriate credit to the original author(s) and the source, provide a link to the Creative Commons license, and indicate if changes were made. The Creative Commons Public Domain Dedication waiver (http://creativecommons.org/publicdomain/zero/1.0/) applies to the data made available in this article, unless otherwise stated. 